\title{
A Refinement of the Square Wave Phase Noise Evaluation
}

\author{
Ludwig Lubich \\ Technical University of Sofia \\ Sofia, Bulgaria \\ lv1@tu-sofia.bg
}

\begin{abstract}
Oscillators are often followed by square wave forming circuits and frequency dividers. Traditionally, the level of the phase noise, transferred from the oscillator outputs to the square waves obtained is calculated ignoring the correlations in the oscillator phase noise spectrum. In this paper, accurate expressions are derived, taking into account the phase noise mechanisms in the oscillators. The phase noise power spectral densities are calculated in both the traditional way and by using the proposed expressions and they are compared. The situations where the proposed expressions can be useful are identified.
\end{abstract}

Keywords-phase noise; square wave; frequency divider; oscillator

\section{INTRODUCTION}

A common communication arrangement consists of an oscillator (usually nearly sinusoidal), whose waveform is converted to rectangular by some kind of square wave forming circuit often followed by digital frequency dividers. An important present-day example is the local oscillator frequency synthesis in highly integrated receivers for cognitive and software radio applications where the wide range of frequencies needed is obtained by relatively narrow range PLLs followed by frequency divider chains [1-3]. The Square Wave (SW) may not physically exist. For example, in the switching mixers [4], the input RF signal is effectively multiplied by a nearly rectangular waveform even if the mixer is driven by a sine oscillator. Usually the Power Spectral Density (PSD) of the oscillator Phase Noise (PN) is known, but the PN of the resulting square wave is what really matters. It is widely agreed [5-8] that the phase noise of the formed square wave, when considered in the time domain, is a sampled version of the oscillator $\mathrm{PN}$, having aliasing as a result. Therefore the SW PN spectrum is a sum of frequency translated replicas of the oscillator PN spectrum. The evaluation of square wave's PN PSD by summing up frequency shifted replicas of the oscillator PN PSD, tacitly assuming that the oscillator phase noise is uncorrelated in the frequency domain, is widely accepted $[5,6,8]$. Such an assumption leads to sufficiently exact results in most cases, but strictly speaking is not correct. If the mechanism of phase noise arising in oscillators is considered, it turns out that the oscillator phase noise is correlated. In this paper, the phase noise PSD of square waves obtained from a sine oscillator by edge forming and frequency division is evaluated, taking into account the phase noise model presented in $[9,10]$. The investigations are restricted to the phase noise transferred from the oscillator to the SW and do not include the phase noise contribution of the noise sources in the SW forming circuits and dividers themselves.

\section{OSCILLATOR PHASE NOISE}

Real-world oscillations have both amplitude and phase noise. However, the inherent amplitude-limiting mechanisms of the practical oscillators strongly suppress the amplitude noise. Therefore the PN is the dominating one [10]. A sinusoidal oscillation with $\mathrm{PN}$ can be expressed as $s(t)=A \sin \left[2 \pi f_{0} t+\phi(t)\right]$, where the excess phase $\phi(t)$ represents the PN. Taking into account that $\phi(t)$ is small, we obtain:

$$
s(t) \approx A \sin \left(2 \pi f_{0} t\right)+\phi(t) A \cos \left(2 \pi f_{0} t\right)
$$

According to the PN model presented in [10], the excess phase $\phi(t)$ in an oscillator can be expressed as:

$$
\phi(t)=\frac{1}{q_{\max }} \int_{-\infty}^{t} \Gamma\left(\omega_{0} \tau\right) i(\tau) d \tau
$$

where $\Gamma\left(\omega_{0} \tau\right)$ is called impulse sensitivity function (ISF) and is a dimensionless, frequency- and amplitude-independent periodic function, $\mathrm{q}_{\max }$ is the maximum charge displacement across the capacitor in the oscillator LC tank and $\mathrm{i}(\mathrm{t})$ is a noise current injected to the LC tank that can model different noise sources in the oscillator [10]. The ISF reflects the sensitivity of the oscillator phase to an impulse injected at phase $\omega_{0}$ t. The ISF can be approximated by Fourier series. Multiplying its terms by the noise current in (2) results in frequency translations of the noise spectrum by integer multiples of the oscillation frequency $f_{0}$. Therefore the same components of the original noise $\mathrm{i}(\mathrm{t})$ are present at different frequencies in the $\mathrm{PN}$ spectrum, thus there are nonzero correlations between the PN spectral components.

\section{PSD OF THE SQUARE WAVE PHASE NOISE}

\section{A. Phase Noise of Non-Sinusoidal Waveforms}

A non-sinusoidal oscillation is a sum of harmonics, each with its own phase noise sidebands which overlap. It is 
impossible to distinguish the phase noise of the fundamental and the phase noise of the other harmonics. But this is not necessary. Indeed the total noise PSD is what matters. Let us consider a periodic non-sinusoidal noiseless waveform $\bar{s}(t)$. Its fundamental is the component of interest and it can be expressed as $A \sin \left(2 \pi f_{W} t\right)$ where A denotes its amplitude. If the corresponding noisy waveform can be expressed as

$$
s(t)=\bar{s}(t)+A \phi(t) \cos \left(2 \pi f_{W} t\right)
$$

then, comparing with (1) we can interpret $\phi(t)$ as phase noise of $s(t)$.

\section{B. Phase Noise After Square Wave Forming}

Although it is intuitively plausible that the square wave forming results in a sampling of the oscillator phase noise in the time domain, it is highly desirable to examine this supposition a bit more rigorously. Let us consider a sinusoidal oscillator with an oscillation frequency $f_{0}$ followed by an SW forming circuit. Without loss of generality, a unity oscillation amplitude is assumed. In the general case, a frequency division by a factor $D$ also takes place along with the SW forming. (For $\mathrm{SW}$ forming without frequency division $\mathrm{D}=1$ ). The $\mathrm{SW}$ has a frequency of $\mathrm{f}_{\mathrm{SW}}=\mathrm{f}_{0} / \mathrm{D}$ and a duty cycle of $1 / 2$. (Other duty cycles are hardly applicable in wireless communications.) The edges of the SW occur around the time instants $k D T_{0} / 2$. The peak-to-peak voltage of the SW is $V_{P P}$. It is easy to establish that the fundamental amplitude of the $\mathrm{SW}$ is $A=2 V_{P P} / \pi$.

The following assumptions will be adopted:

1. The edge forming of the pulses can be adequately modeled by passing the oscillator sine wave via limiting amplifiers with an unsaturated voltage gain $\alpha$ or $-\alpha$.

2. The edge duration $t_{e}$ of the $\mathrm{SW}$ is at least an order of magnitude shorter than the oscillator period.

3. The phase disturbance $\phi_{O S C}(t)$ at the oscillator output is nearly constant in time intervals $\Delta t \leq \phi_{O S C}(t) /\left(2 \pi f_{0}\right)$.

4. The time extents $\Delta t=\phi_{O S C}(t) /\left(2 \pi f_{0}\right)$ are orders of magnitude shorter than the edge duration.

The first assumption can be replaced by another one: The edge forming circuit has a perfect threshold behavior and the oscillator PN affects the edge positions of the SW instantaneously at the oscillation zero-crossing instants only, shifting the edges by $\phi_{O S C}\left(k D T_{0} / 2\right) / \omega_{0}$. Then the obtained PN PSD of the SW for the frequencies of interest becomes the same as the PN PSD obtained here. Obviously, the oscillator PN can affect the SW during the edges only. This can be modeled by multiplying the excess phase $\phi_{O S C}$ by corresponding time shifted pulses $p(t)$ with a unity amplitude and duration $\mathrm{t}_{\mathrm{e}}$.

The oscillator waveform is given by:

$$
\begin{aligned}
v_{O S C}(t) & \approx \sin \left(2 \pi f_{0} t\right)+\phi_{O S C}(t) \cos \left(2 \pi f_{0} t\right) \\
& =\sin \left(2 \pi f_{0} t\right)+n_{O S C}(t)
\end{aligned}
$$

Let the rising edges of the SW occur when $v_{O S C}(t)$ rises, i.e. at $t=n D T_{0}$ where $T_{0}=1 / f_{0}$ is the oscillator period and $\mathrm{n}$ is an arbitrary integer. Then the falling edges occur at $t=n D T_{0}+D T_{0} / 2$. Hence if the frequency division factor $\mathrm{D}$ is odd, the falling edges occur when $v_{O S C}(t)$ falls; if $\mathrm{D}$ is even, then the falling edges of the SW also occur when $v_{O S C}(t)$ rises (Figure 1).

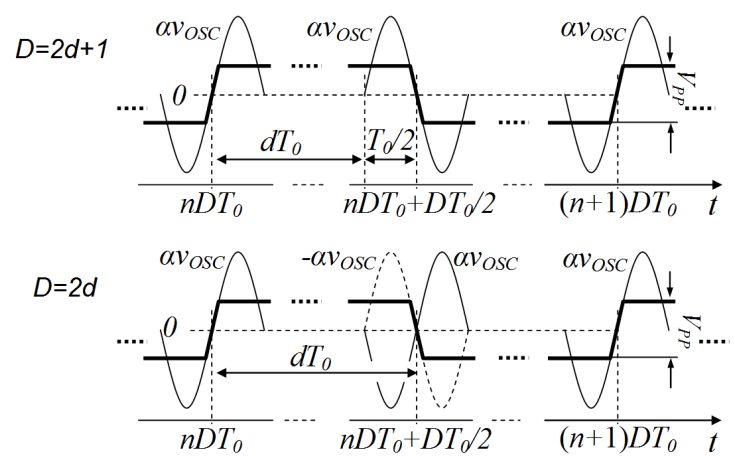

Fig. 1. Sine to rectangle conversion for odd and even values of the frequency division ratio $\mathrm{D}$

Therefore, taking assumption 1 into account, the SW during the k-th edge can be expressed as:

$$
v_{S W}(t)=\left[(-1)^{D+1}\right]^{k} \alpha v_{\text {OSC }}(t)
$$

Then the noise during the k-th edge can be expressed as

$$
n_{k}(t)=(-1)^{k(D+1)} \alpha n_{O S C}(t)
$$

For the k-th edge, taking into account assumption 2, we can approximate the noise term of $v_{O S C}(t)$ as:

$$
n_{O S C}(t) \approx \phi_{O S C}(t) \cos \left(2 \pi f_{0} k D T_{0} / 2\right)=\phi_{O S C}(t)(-1)^{k D}
$$

Then after substituting (7) in (6), we obtain:

$$
n_{k}(t)=(-1)^{k(D+1)} \alpha \phi_{O S C}(t)(-1)^{k D}=(-1)^{k} \alpha \phi_{O S C}(t)
$$

The total noise of the SW can be obtained by summing up the noise of all SW edges, multiplied by the corresponding gating pulses:

$$
n_{S W}(t)=\sum_{k=-\infty}^{\infty} n_{k}(t) p\left(t-k \frac{D T_{0}}{2}\right)
$$

Substituting (8) in (9) and taking into account assumption 2, we can express (9) as:

$$
\begin{gathered}
n_{S W}(t) \approx \alpha \cos \left(2 \pi f_{S W} t\right) \varphi_{\text {OSC }}(t) . \\
\sum_{k=-\infty}^{\infty} p\left(t-k \frac{D T_{0}}{2}\right)
\end{gathered}
$$


After inspecting (10) and comparing it with the noise term in (3), we can recognize that the noise of the SW is phase noise only. To obtain the SW excess phase $\phi_{S W}(t)$, we bring (10) to the form $n_{S W}(t)=A \cos \left(2 \pi f_{S W} t\right) \phi_{S W}(t)$ where $A=2 V_{P P} / \pi$ is the fundamental amplitude of the SW. Then

$$
\phi_{S W}(t)=\frac{\alpha \pi}{2 V_{P P}} \phi_{O S C}(t) \sum_{k=-\infty}^{\infty} p\left(t-k \frac{D T_{0}}{2}\right)
$$

Equation (11) shows that in the time domain the SW PN is the oscillator PN non-ideally sampled at a rate of $2 f_{O S C} / D=2 f_{S W}$ and scaled by magnitude. Taking into account assumptions 1 and 2, it can be established that $V_{P P} \approx \alpha 2 \pi f_{0} t_{e}$. Then

$$
\phi_{S W}(t) \approx \frac{T_{0}}{4 t_{e}} \phi_{O S C}(t) \sum_{k=-\infty}^{\infty} p\left(t-k \frac{D T_{0}}{2}\right)
$$

\section{Evaluating the Square Wave Phase Noise PSD}

The PN PSD is found as

$$
S_{S W}(f)=\lim _{T \rightarrow \infty} \frac{\mathrm{E}\left[\left|\dot{\Phi}_{S W}(f)_{T}\right|^{2}\right]}{T}
$$

where $\dot{\Phi}_{S W}(f)$ is the Fourier transform of $\phi_{S W}(t)$, the subscript $\mathrm{T}$ denotes a truncation to a finite time interval $\mathrm{T}$ and $\mathrm{E}[X]$ denotes expectation [11]. Taking the Fourier transform of $\phi_{S W}(t)$, we obtain:

$$
\dot{\Phi}_{S W}(f)_{T}=\frac{1}{2 D t_{e}} \sum_{k=-\infty}^{\infty} \dot{P}\left(2 k f_{S W}\right) \dot{\Phi}_{O S C}\left(f-2 k f_{S W}\right)_{T}
$$

where $\dot{P}(f)$ and $\dot{\Phi}_{O S C}(f)_{T}$ are the Fourier transforms of $p(t)$ and $\phi_{O S C}(t)$, respectively. It can be seen that $\dot{\Phi}_{S W}(f)_{T}$ is a sum of replicas of $\dot{\Phi}_{O S C}(f)_{T}$ shifted by integer multiples of $2 f_{S W}$. Assuming that $\dot{\Phi}_{O S C}(f)_{T}$ is not correlated in the frequency domain, the PN PSD can be found as:

$$
S_{S W}=\left(\frac{1}{2 D t_{e}}\right)^{2} \sum_{k=-\infty}^{\infty}\left|\dot{P}\left(2 k f_{S W}\right)\right|^{2} S_{O S C}\left(f-2 k f_{S W}\right)
$$

where $S_{O S C}(f)$ denotes the PSD of the oscillator PN. Equation (15) expresses the traditional way of evaluating the PN PSD. However, $\dot{\Phi}_{O S C}(f)_{T}$ is correlated. In the oscillator, each component of the spectrum of the original noise $\mathrm{i}(\mathrm{t})$ undergoes frequency translations by multiples of $f_{0}$, as it was discussed above. In the general case, this includes frequency translations differing by multiples of $2 f_{S W}$ from each other. Next, the aliasing due to the sampling causes further frequency translations of the oscillator PN spectrum by multiples of $2 f_{S W}$. Therefore, each component of the original noise will get to the same frequencies in many (strictly speaking countless) different ways, as illustrated in Fig. 2. Then the voltages of these replicas of the same noise component should be summed up (instead their powers). In contrast, in (15) only power summations are performed.

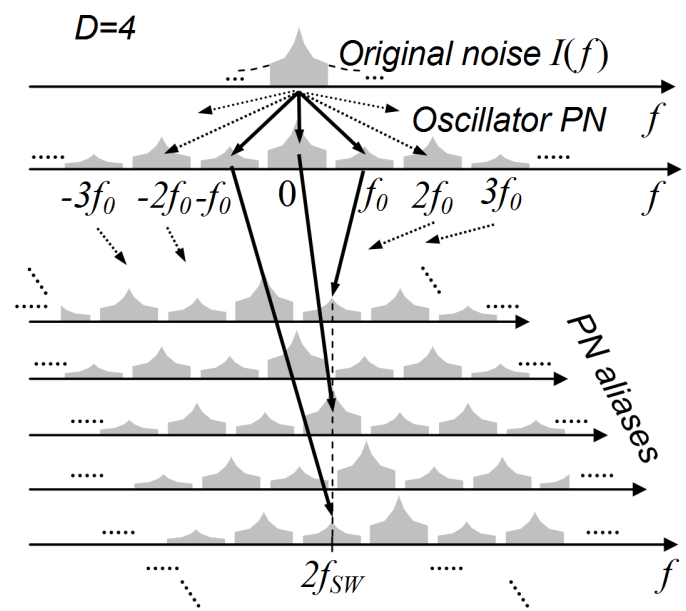

Fig. 2. Frequency translations for $D=4$. For the sake of clarity, only three different ways for only one frequency translation (by $2 f_{S W}$ ) are indicated by solid arrows and the magnitude decay caused by the integration is not presented.

Let us express $\dot{\Phi}_{O S C}(f)_{T}$ following the oscillator PN model presented in Section II. We will deviate a little from the original designations of [10]: The ISF will be an explicit function of the time, periodic in $T_{0}=1 / f_{0}$ and will be designated by $\gamma(\mathrm{t})$. Its Fourier transform will be denoted by $\Gamma(f)$. Similarly, the Fourier transform of the original noise current $\mathrm{i}(\mathrm{t})$ will be denoted by $I(f)$. Thus, the phase disturbances are expressed by

$$
\phi_{O S C}(t)=\frac{1}{q_{\max }} \int_{-\infty}^{t} \gamma(\tau) i(\tau) d \tau
$$

Then, taking Fourier transform of (16) and substituting in (14), we obtain:

$$
\begin{aligned}
\dot{\Phi}_{S W}(f)_{T}= & \frac{1}{q_{\max }} \frac{1}{j 4 \pi D t_{e}} \\
& \times \sum_{k=-\infty}^{\infty} \frac{\dot{P}\left(2 k f_{S W}\right)}{f-2 k f_{S W}} \sum_{n=-\infty}^{\infty} \dot{C}_{n} I\left[f-f_{S W}(2 k+n D)\right]_{T}
\end{aligned}
$$

where $\dot{C}_{n}$ are the complex Fourier series coefficients of the ISF. The ISF can be well approximated by a limited number of Fourier series terms, i.e. $n$ can be restricted to a certain range $\left[-n_{\max }, n_{\max }\right]$.

If $D$ is an even number, i.e. $D=2 d$, (17) can be expressed as:

$$
\begin{aligned}
\dot{\Phi}_{S W}(f)_{T}= & \frac{1}{q_{\max }} \frac{1}{j 4 \pi D t_{e}} \\
& \times \sum_{k=-\infty}^{\infty} \frac{\dot{P}\left(2 k f_{S W}\right)}{f-2 k f_{S W}} \sum_{n=-n_{\max }}^{n_{\max }} \dot{C}_{n} I\left[f-2 f_{S W}(k+n d)\right]_{T}
\end{aligned}
$$


After making the substitution $l=k+n d$ and simplifying we obtain:

$$
\begin{aligned}
\dot{\Phi}_{S W}(f)_{T}= & \frac{1}{q_{\max }} \frac{1}{j 4 \pi D t_{e}} \\
& \times \sum_{l=-\infty}^{\infty} I\left[f-2 l f_{S W}\right]_{T} \sum_{n=-n_{\max }}^{n_{\max }} \frac{\dot{C}_{n} \dot{P}\left[(2 l-n D) f_{S W}\right]}{f-(2 l-n D) f_{S W}}
\end{aligned}
$$

The second sum in (19) contains the scaling factors of all original noise spectrum replicas experiencing the same frequency translation. Taking into account the roll-off caused by the integration, it can be established that a very good accuracy for the frequency range of interest can be preserved if a constant $\dot{P}(f) \approx \dot{P}(0)=t_{e}$ is assumed. Then (19) is simplified to:

$$
\begin{aligned}
\dot{\Phi}_{S W}(f)_{T} \approx & \frac{1}{q_{\max }} \frac{1}{j 4 \pi D} \\
& \times \sum_{l=-\infty}^{\infty} I\left(f-2 l f_{S W}\right)_{T} \sum_{n=-n_{\max }}^{n_{\max }} \frac{\dot{C}_{n}}{f-(2 l-n D) f_{S W}} .
\end{aligned}
$$

After substituting (20) in (13) and simplifying, we obtain the PSD of the SW PN:

$$
S_{S W}=K^{2} \sum_{l=-\infty}^{\infty} S_{i}\left(f-2 l f_{S W}\right)\left|\sum_{n=-n_{\max }}^{n_{\max }} \frac{\dot{C}_{n}}{f-(2 l-n D) f_{S W}}\right|^{2}
$$

where $S_{i}$ denotes the PSD of the original noise $i(t)$ and $K=\left(4 \pi D q_{\max }\right)^{-1}$. If $D=1,(17)$ can be expressed as :

$\dot{\Phi}_{S W}(f)_{T} \approx \frac{K}{j} \sum_{k=-\infty}^{\infty} \sum_{m=-m_{\max }}^{m_{\max }}\left\{\begin{array}{l}\frac{\dot{C}_{2 m} I\left[f-(2 k+2 m) f_{0}\right]_{T}}{f-2 k f_{0}} \\ +\frac{\dot{C}_{2 m+1} I\left[f-(2 k+2 m+1) f_{0}\right]_{T}}{f-2 k f_{0}}\end{array}\right\}$

After making the substitution $\mathrm{k}+\mathrm{m}=1$, we obtain:

$$
\dot{\Phi}_{S W}(f)_{T} \approx \frac{K}{j}\left\{\begin{array}{l}
\sum_{l=-\infty}^{\infty} I\left(f-2 l f_{0}\right)_{T} \sum_{m=-m_{\max }}^{m_{\max }} \frac{\dot{C}_{2 m}}{f-2(l-m) f_{0}} \\
+\sum_{l=-\infty}^{\infty} I\left[f-(2 l+1) f_{0}\right]_{T} \sum_{m=-m_{\max }}^{m_{\max }} \frac{\dot{C}_{2 m+1}}{f-2(l-m) f_{0}}
\end{array}\right\}
$$

After substituting (23) in (13) and simplifying we obtain the PN PSD:

$$
S_{S W}=K^{2}\left\{\begin{array}{l}
\sum_{l=-\infty}^{\infty} S_{i}\left(f-2 l f_{0}\right)\left|\sum_{m=-m_{\max }}^{m_{\max }} \frac{\dot{C}_{2 m}}{f-2(l-m) f_{0}}\right|^{2} \\
+\sum_{l=-\infty}^{\infty} S_{i}\left[f-(2 l+1) f_{0}\right]\left|\sum_{m=-m_{\max }}^{m_{\max }} \frac{\dot{C}_{2 m+1}}{f-2(l-m) f_{0}}\right|^{2}
\end{array}\right\}
$$

The splitting of (24) in two terms reflects the obvious fact that the total frequency translation $(2 k+n) f_{0}$ of the original noise PSD can be an even multiple of $f_{0}$ for even values of $n$ only and an odd multiple of $f_{0}$ for odd values of $n$. In a similar way, an expression for SW phase noise PSD for arbitrary odd values of D can be derived. It is not shown here, because odd division ratios are rarely used. At this point the PSD of $\phi_{S W}(t)$ is known. However, we need to know the PSD of the PN sidebands around the SW fundamental. The multiplication of the excess phase by the cosine in (3) produces a sum of two PN spectrum replicas shifted by $+f_{S W}$ and $-f_{S W}$, or shifted by $2 f_{S W}$ from each other. Since the PN has a sampling rate of $2 f_{S W}$, the PN spectrum has a periodicity of $2 f_{S W}$, therefore the two shifted PN spectrum replicas are absolutely identical. Hence, its summation results in a doubled noise voltage and thus the resultant PSD becomes 4 times higher than $S_{S W}$.

\section{SimULATION RESULTS}

Equations (21) and (24) were verified by numerous Matlab simulations. The discrepancy between the calculated PSD and the simulation results was less than one tenth of a decibel. The use of the approximation $\dot{P}(f) \approx t_{e}$ increased the prediction error to roughly $1 \mathrm{~dB}$ for relatively long edge durations. It has been observed that when the ISF has only a dc component (a purely hypothetical ISF), there is no difference between the PSD obtained using the proposed expressions and the PSD calculated in the traditional way. Conversely, when the ISF has at least one $\mathrm{AC}$ component, the traditional calculation gives nonzero errors. This is in agreement with our theory, because each frequency translation of the original noise happens in a unique way in the first case, while in the second case the original noise undergoes the same frequency translations in two or more different ways. The simulations showed that the error of the traditional calculation reaches a few decibels when the flicker noise corner $f_{1 / f}$ of the original noise $i(t)$ is higher than several tens of percent of the oscillation frequency. Errors of the traditional calculation of up to several decibels were observed when the original noise PSD showed steep changes, but this is unlikely for a real-world noise source. It was observed in all simulations that for frequency offsets up to tens of percent of the oscillator frequency the error of the traditional calculation is negligible (below one tenth of a decibel).

\section{SOME EXAMPLES}

First we will examine a usual case: SW forming with a frequency division ratio of two. The example ISF is given by: $1+2 \cos \left(2 \pi f_{0} t\right)$. The following parameters are chosen: original noise PSD of $-160 \mathrm{dBm} / \mathrm{Hz}$ for $f>f_{1 / f}$, oscillator frequency $f_{0}=200 \mathrm{MHz}$ and maximum charge displacement across the capacitor in the oscillator LC tank $q_{\max }=1.5 \cdot 10^{-11} \mathrm{C}$. The flicker noise corner frequency is $f_{1 / f}=10 \mathrm{MHz}$. The flicker noise corners in this and in following examples may seem to be unrealistically high, but in the contemporary nanoscale CMOS processes $f_{1 / f}$ can be well beyond $1 \mathrm{MHz}$ [12] and can even reach hundreds of megahertz $[13,14]$. The PN PSDs calculated by the proposed expression (21) and obtained by simulation are shown in Figure 3. A perfect agreement between the calculated and simulated PSD can be seen. The PSD calculated in the 
traditional way (15) is also shown. Practically there is no difference between two calculated PSDs. In Figure 4 the results for $f_{1 / f}=200 \mathrm{MHz}$ are given, with other conditions unchanged. The error of the traditional calculation (15) reaches nearly $2 \mathrm{~dB}$ for frequency offsets approaching $f_{0}$, while the PSD calculated by (21) remains close to the simulated PSD. However, the PN PSD for such offsets is usually well below the thermal noise PSD and the total noise will be dominated by the thermal and the device noise of the divider circuits. Therefore, the error caused by the simplified calculation will be ultimately negligible again.

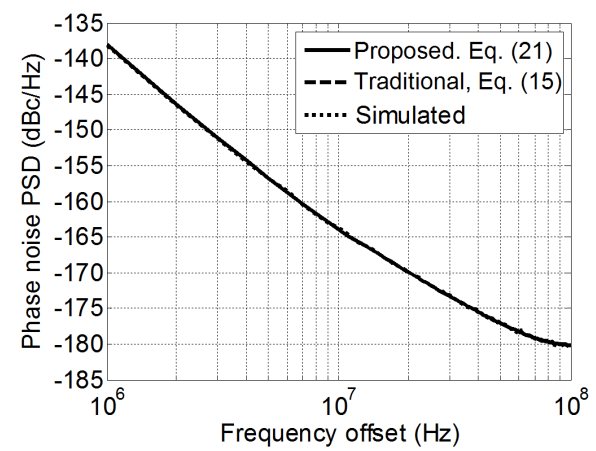

Fig. 3. Comparison between the calculated PN PSD and the PN PSD obtained by simulation for $f_{0}=200 \mathrm{MHz}, D=2$ and $f_{1 / f}=10 \mathrm{MHz}$.
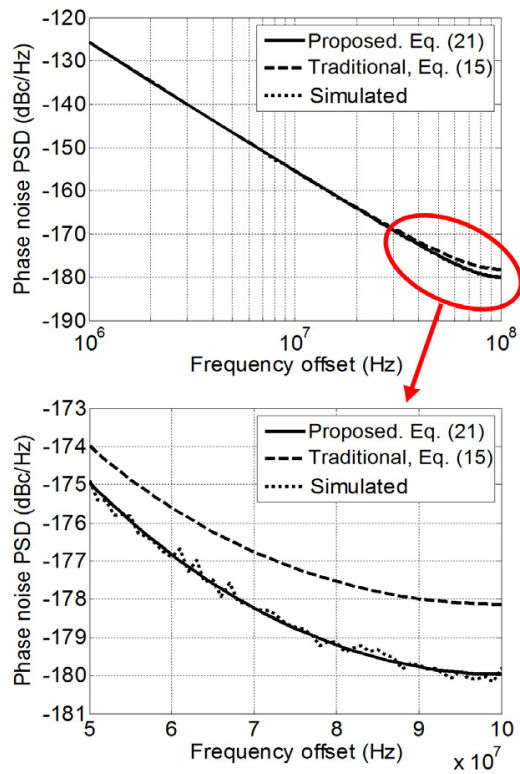

Fig. 4. Comparison between the calculated PN PSD and the PN PSD obtained by simulation for $f_{0}=200 \mathrm{MHz}, D=2$ and $f_{1 / f}=200 \mathrm{MHz}$.

Let us consider next an example for SW forming without frequency division. The oscillator frequency is $f_{0}=100 \mathrm{MHz}$. The ideal ISF [10], i.e. $\gamma(t)=\cos \left(2 \pi f_{0} t\right)$, is chosen. The other conditions are the same as in the first example. The calculation and simulation results are given in Figure 5. The traditional calculation erroneously predicts a non-existing steep upsurge of the PSD when the frequency offset approaches $f_{0}$. The error goes beyond $6 \mathrm{~dB}$, while the PSD calculation according to the proposed formula (24) gives correct results. As in the previous example, the error increases when the flicker noise corner is higher.
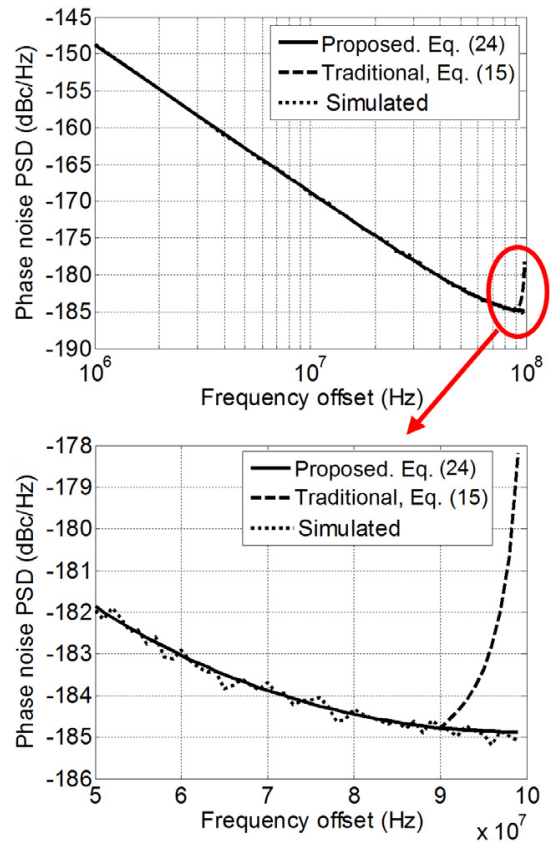

Fig. 5. Comparison between the calculated PN PSD and the PN PSD obtained by simulation for $f_{0}=100 \mathrm{MHz}, D=1$ and $f_{1 / f}=10 \mathrm{MHz}$.

\section{CONCLUSIONS}

A faultless calculation of the square wave phase noise PSD cannot be performed if the oscillator and SW forming circuits are not considered as one whole. Taking into account the phase noise mechanism in the oscillators, expressions for exact calculation of SW phase noise PSD were derived. A practically perfect agreement between the calculated and the simulated PSDs was obtained. However, in most practical cases, especially at relatively low frequency offsets, the error of the widely used simplified calculation is insignificant. The proposed expressions may be useful when the PN PSD at large frequency offsets is important and at the same time one or more of the following are present: the PSD of the oscillator noise sources is relatively high, the flicker noise corner of the oscillator noise sources is higher than several tenths of the oscillation frequency, there are steep changes in the PSD of the oscillator noise sources. In such cases, an accuracy improvement of several decibels can be obtained using the proposed formulas.

\section{REFERENCES}

[1] H. M. El Misilmani, M. Y. Abou-Shahine, Y. Nasser, K. Y. Kabalan, "Recent Advances on Radio-Frequency Design in Cognitive Radio", International Journal of Antennas and Propagation, Vol. 2016, Article ID 9878475, 2016 
[2] Z. El Alaoui Ismaili, W. Ajib, F. Gagnon, F. Nabki, "Very Wide Range Frequency Synthesizer Architecture for Avionic SDR Applications", IEEE International Conference on Ubiquitous Wireless Broadband, pp. $1-5,2015$

[3] B. Razavi, "Challenges in the Design of Cognitive Radios", IEEE Custom Intergrated Circuits Conference, pp. 391-398, 2009

[4] B. Razavi, RF Microelectronics, Prentice Hall PTR, 2011

[5] C. Calosso, E. Rubiola, "The Sampling Theorem in Pi and Lambda Digital Frequency Dividers", Joint UFFC, EFTF and PFM Symposium, pp. 960-962, 2013

[6] M. Apostolidou, P. Baltus, C. Vaucher, "Phase Noise in Frequency Divider Circuits", IEEE International Symposium on Circuits and Systems, pp. 2538-2541, 2008

[7] S. Levantino, L. Romano, S. Pellerano, C. Samori, A. L. Lacaita, "Phase Noise in Digital Frequency Dividers", IEEE Journal of Solid-State Circuits, Vol. 39, No. 5, pp. 775-784, 2004

[8] W. F. Egan, "Modeling phase noise in frequency dividers", IEEE Transactions on Ultrasonics, Ferroelectrics, and frequency Control, Vol. 37, No. 4, pp. 307-315, 1990
[9] T. H. Lee, A. Hajimiri, The Design of Low Noise Oscillators, Kluwer Academic Publishers, 2003

[10] T. H. Lee, A. Hajimiri, "Oscillator Phase Noise: A Tutorial", IEEE Journal of Solid-State Circuits, Vol. 35, No. 3, pp. 326-336, 2000

[11] Д. В. Васильев, М. Р. Витоль, Ю. Н. Горшенков, К. Самойло, Т. Федосова, Е. Черниговская, Радиотехнические цепи и сигналы [Radiotechnical circuits and signals], Радио и связь, Москва, 1982 [In Russian]

[12] F. Pepe, A. Bonfanti, S. Levantino, C. Samori, A. L. Lacaita, "Suppression of Flicker Noise Up-Conversion in a 65-nm CMOS VCO in the 3.0-to-3.6 GHz Band", IEEE Journal of Solid-State Circuits, Vol. 48, No. 10, pp. 2375-2389, 2013

[13] I. Chlis, D. Pepe, D. Zito, "Analyses and techniques for phase noise reduction in CMOS Colpitts oscillator topology", International Journal of Circuit Theory and Applications, Vol. 44, No. 3, pp. 616-638, 2016

[14] I. Chlis, D. Pepe, D. Zito, "Analysis of Phase Noise in $28 \mathrm{~nm}$ CMOS LC Oscillator Differential Topologies: Armstrong, Colpitts, Hartley and Common-Source Cross-Coupled Pair" Journal of Circuits, Systems, and Computers, Vol. 24, No. 4, pp. 1550052-1 - 1550052-18, 2015 